\title{
Creation of problem-dependent Doppler-broadened cross sections in the KENO Monte Carlo code
}

\author{
Shane W.D. Hart ${ }^{\mathrm{a}, *}$, Cihangir Celik ${ }^{\mathrm{a}}$, G. Ivan Maldonado ${ }^{\mathrm{b}}$, Luiz Leal ${ }^{\mathrm{a}}$ \\ ${ }^{a}$ Oak Ridge National Laboratory \\ ${ }^{b}$ The University of Tennessee
}

\begin{abstract}
This paper introduces a quick method for improving the accuracy of Monte Carlo simulations by generating one- and twodimensional cross sections at a user-defined temperature before performing transport calculations. A finite difference method is used to Doppler-broaden cross sections to the desired temperature, and unit-base interpolation is done to generate the probability distributions for double differential two-dimensional thermal moderator cross sections at any arbitrarily user-defined temperature. The accuracy of these methods is tested using a variety of contrived problems. In addition, various benchmarks at elevated temperatures are modeled, and results are compared with benchmark results. The problem-dependent cross sections are observed to produce eigenvalue estimates that are closer to the benchmark results than those without the problem-dependent cross sections.
\end{abstract}

Keywords: Monte Carlo, Doppler broadening, thermal scattering, KENO, SCALE

\section{Introduction}

Cross-section files are generally provided in Evaluated Nuclear Data Format (ENDF) formatted data files [1] that contain all of the necessary data to create continuous en5 ergy (CE) data libraries for use in a Monte Carlo calculation. To be useful, these ENDF data files are generally processed by a cross-section processing code such as AMPX [2] or NJOY [3] for use in a radiation transport code such as CE-KENO 4. For one-dimensional cross sections, the 10 data are usually provided at one temperature (designated as $0 \mathrm{~K}$ ), and need to be Doppler-broadened to various temperatures before they can be used at reactor-level temperatures. [

Exact Doppler-broadened cross sections can be done by 15 the nuclear data-processing codes using Doppler broadening equations [5]; however, producing exact cross sections at a large number of temperatures would consume a significant amount of time and space, both in memory and on a

\footnotetext{
* Corresponding author

Email addresses: hartsw@ornl.gov (Shane W.D. Hart), celikc@ornl.gov (Cihangir Celik), imaldona@utk.edu (G. Ivan Maldonado), leall@ornl.gov (Luiz Leal)

This manuscript has been authored by UT-Battelle, LLC under Contract No. DE-AC05-00OR22725 with the U.S. Department of Energy. The United States Government retains and the publisher, by accepting the article for publication, acknowledges that the United States Government retains a non-exclusive, paid-up, irrevoca- ${ }^{45}$ ble, world-wide license to publish or reproduce the published form of this manuscript, or allow others to do so, for United States Government purposes. The Department of Energy will provide public access to these results of federally sponsored research in accordance with the DOE Public Access Plan (http://energy.gov/downloads/doe-publicaccess-plan).
}

hard disk. Therefore, cross-section libraries are generally only created at several different temperatures; for KENO, part of the SCALE code suite [6], there are generally six temperatures created. For KENO in CE mode, if the temperature desired is not one of the pregenerated temperatures, then the closest temperature is used. A case containing materials that are $50 \mathrm{~K}$ away from a library temperature can produce significantly different results when compared with a case that is using the temperature-corrected cross sections.

Two-dimensional cross sections are generally provided for thermal moderators in order to account for crystalline effects encounted when neutrons are traveling at thermal speeds. Unlike the one-dimensional cross sections, the ENDF files are usually provided at a variety of temperatures. However, no Doppler broadening is done on these temperatures, so the end result is the same: If a temperature desired by the user is sufficiently far from the library temperatures, errors in the eigenvalue estimates can occur. Some previous work has been done to provide for on-thefly (OTF) Doppler broadening of one-dimensional neutron cross sections [7, 8, 9, 10, 11 in other Monte Carlo codes. For example, MCNP6 [12] ships with a utility to generate fits to cross-section data so that cross sections can be calculated on-the-fly for any temperature as desired. KENO previously had no such capability to Doppler broaden cross sections.

In this paper two methods are discussed to temperaturecorrect the provided cross sections. A finite difference method is employed for the one-dimensional cross sections. This method is much faster than the exact Dopplerbroadening method developed by Cullen and can use the 
data libraries that have already been created. For twodimensional thermal moderator data, a simple unit-base interpolation scheme is used on the probability distributions of the double differential cross sections. By com- 90 55 bining the aforementioned methods with temperature interpolation on the probability tables covering the unresolved resonance range (such as in 13]), KENO will have temperature-corrected neutron cross sections for all energy regions of interest [14.

\section{2. One-Dimensional Method}

For one-dimensional cross sections the approach to be implemented into KENO utilizes a finite-difference method similar to that used by SAMMY 15, which is well suited for resonance analysis and light water reactor (LWR) applications. This approach is based on the Leal-Hwang scattering method [16], in which the Doppler broadened cross sections satisfy a heat equation of the form

$$
\frac{\partial^{2} F}{\partial u^{2}}=\frac{\partial F}{\partial \zeta}
$$

where $F$ is the function of interest (in this case the cross section), $u$ is the energy range, and $\zeta$ is the temperature. Then, because of the initial condition $F(u, 0)$ for $-\infty<$ $u<\infty$ and the boundary conditions $F(\infty, \zeta)=F(\infty, 0)$ and $F(-\infty, \zeta)=F(-\infty, 0)$, the function $F$ can be calculated using the finite-difference method.

The application of the finite-difference method solves Eq. (1) by applying an explicit finite-difference formalism assuming constant meshes with $\delta u \equiv h$ and $\delta \zeta \equiv \gamma$. The first and second derivatives can then be expanded in a Taylor's series. Thus the explicit finite-difference equation for the function $F$ at any $u_{i}$ and $\zeta_{j+1}$ is

$$
F_{i}^{j+1}=s\left(F_{i+1}^{j}+a F_{i}^{j}+F_{i-1}^{j}\right)
$$

where $s \equiv \frac{\gamma}{h^{2}}$ and $a \equiv \frac{1-2 s}{s}$. The finite-difference equation in Eq. (2) can be modified for nonuniform meshes as

$$
F_{i}^{j+1}=s\left(a F_{i}^{j}+\frac{2\left(F_{i-1}^{j} \delta v_{r}+F_{i+1}^{j} \delta v_{l}\right)}{\delta v_{r}+\delta v_{l}}\right),
$$

where $s \equiv \frac{\gamma}{\delta v_{l} \delta v_{r}}, a \equiv \frac{1-2 s}{s}, \delta v_{l}=v_{i}-v_{i-1}, \delta v_{r}=v_{i+1}-v_{i}$, and $v_{i}$ is the momentum at the $i^{\text {th }}$ grid point.

Selecting a $\Delta T$ that is small (such as $1 \mathrm{~K}$ ) allows for agreement within $0.1 \%$ to reference cross sections across all energies except for very low and very high energies. The increase in error at the edges of the energy range is due to limitations in the finite-difference method. Because each element in the energy grid uses the surrounding elements ${ }_{100}$ to calculate the new value, the finite-difference method produces poor cross-section estimates when surrounding elements are inaccurate or do not exist. In an attempt to alleviate these errors, extra points can be added past the known energy range. This reduces the error for cross $_{105}$ sections at very high and very low energies by extrapolating the known cross-section data but does not eliminate it completely.

Doppler broadening is controlled by the dbx parameter in KENO. By setting dbx to 1, the finite difference approach is enabled. Fig. 1 show the results of using the finite-difference method to obtain cross sections for the ${ }^{238} \mathrm{U}$ scattering reaction at $900 \mathrm{~K}$ near the low-energy tail. As previously discussed, the error in the tail region quickly disappears as one moves away from the energy boundary.

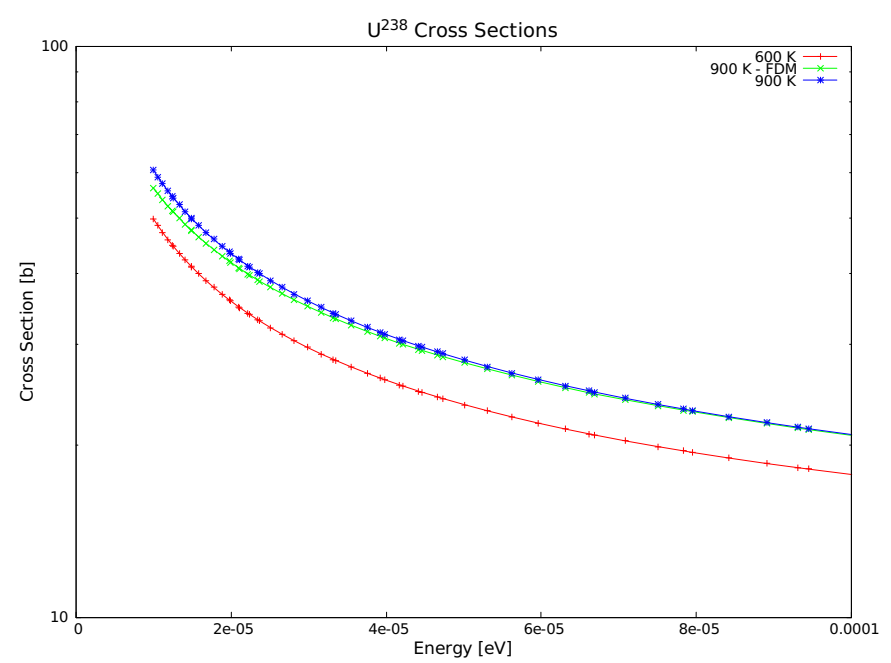

(a) Cross Sections

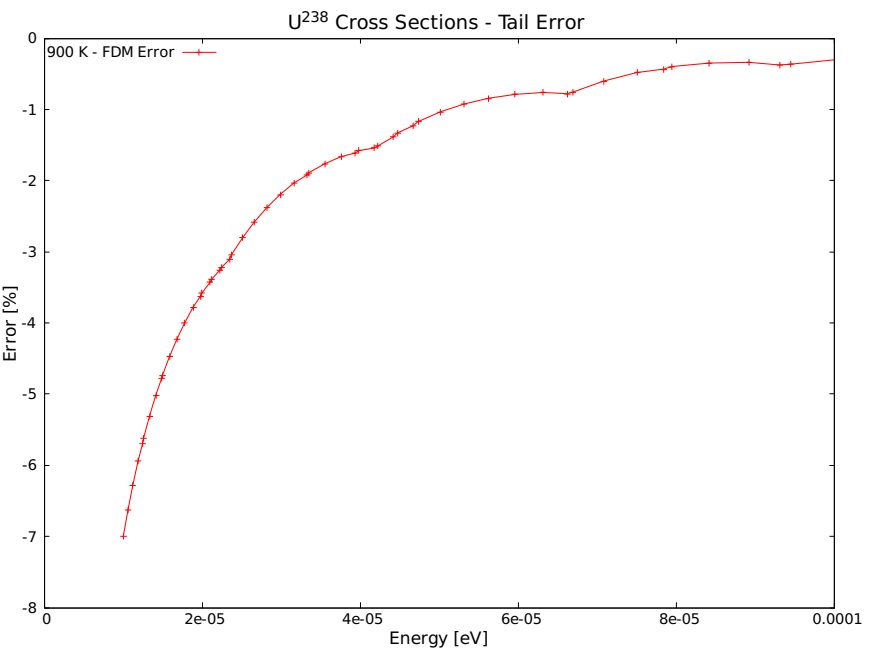

(b) Error

Figure 1: ${ }^{238} \mathrm{U}$ scattering cross sections showing a tail.

As seen in Fig. 1, the error at the low-energy tail can approach $10 \%$. Although the error probably has little effect on the results, it would be beneficial to try and minimize it. One solution is to use linear interpolation for the first five momentum points on the energy grid. Since there are no resonances in this extremely small energy range, doing so would not introduce any errors into the broadened cross sections. By using interpolation for the first ten points, the results from Fig. 1 using the FDM con- 
verge onto $900 \mathrm{~K}$ reference results.

An example of this approach is shown in Fig. 2 for ${ }^{16} \mathrm{O}$. In Fig. 2a there is a large error (approaching 10\%) in the lower tail region. This contrasts sharply with the lack of error in the rest of the energy space, although there is a small error when the finite difference method is used in the resonances. In Fig. $2 \mathrm{~b}$ the error has mostly disappeared and is less than $0.2 \%$ in the tail region. The rest of the energy range is mostly unaffected by the change.

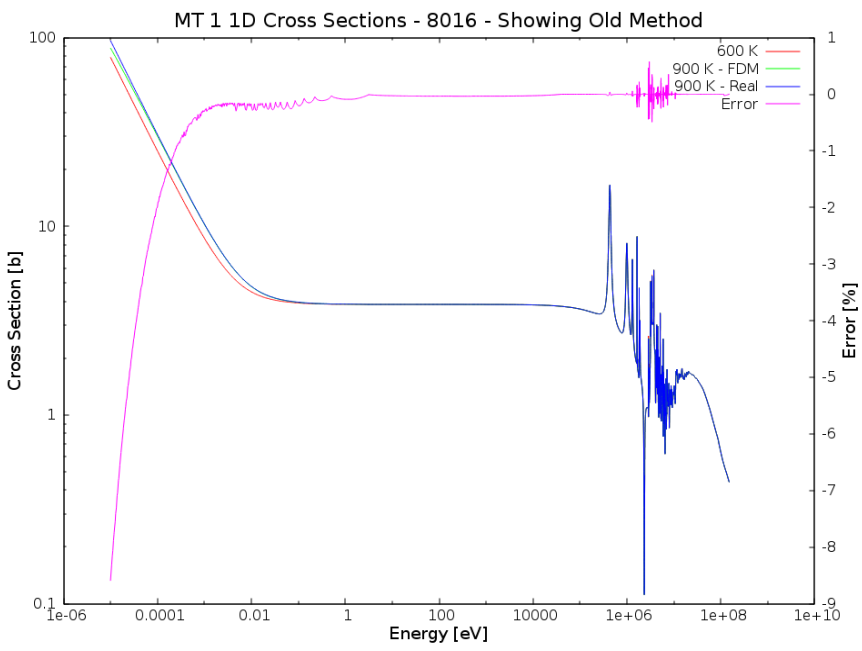

(a) No interpolation on first five points.

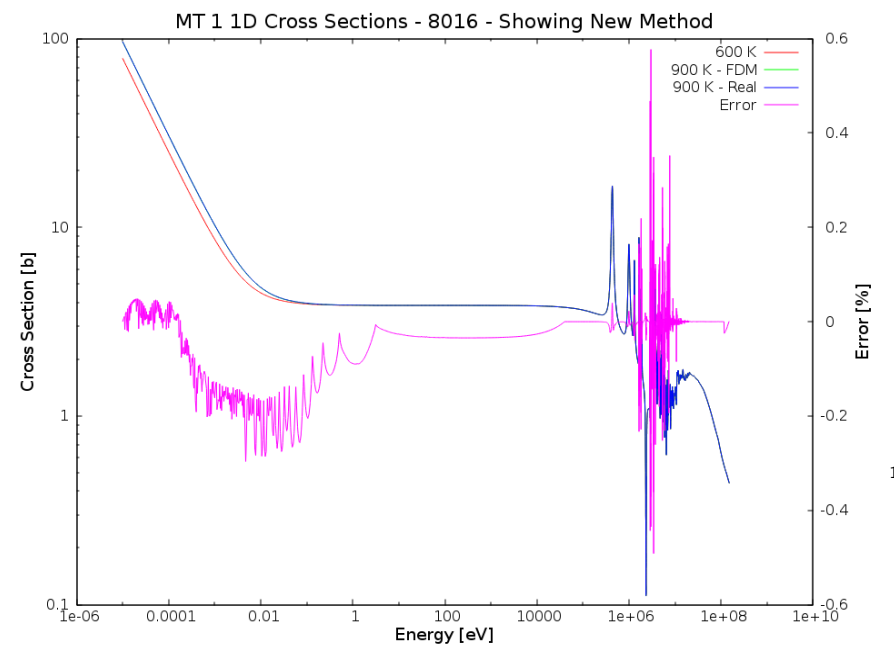

(b) Interpolation on first five points.

Figure 2: Differences in ${ }^{16} \mathrm{O}$ cross sections.

Another problem may arise when dealing with isotopes that do not have a large resonance structure, are light, or have some other small irregularites. One example is ${ }^{1} \mathrm{H}$ in water, which contains no resonances and also isn't smoothly decreasing around $0.01 \mathrm{eV}$. This slight bump causes an instability in the finite difference method as it propagates to the lower energies. This can be seen in Fig. 3a, where the Doppler-broadened cross sections actually exceed the higher-temperature cross sections for the low-energy range.

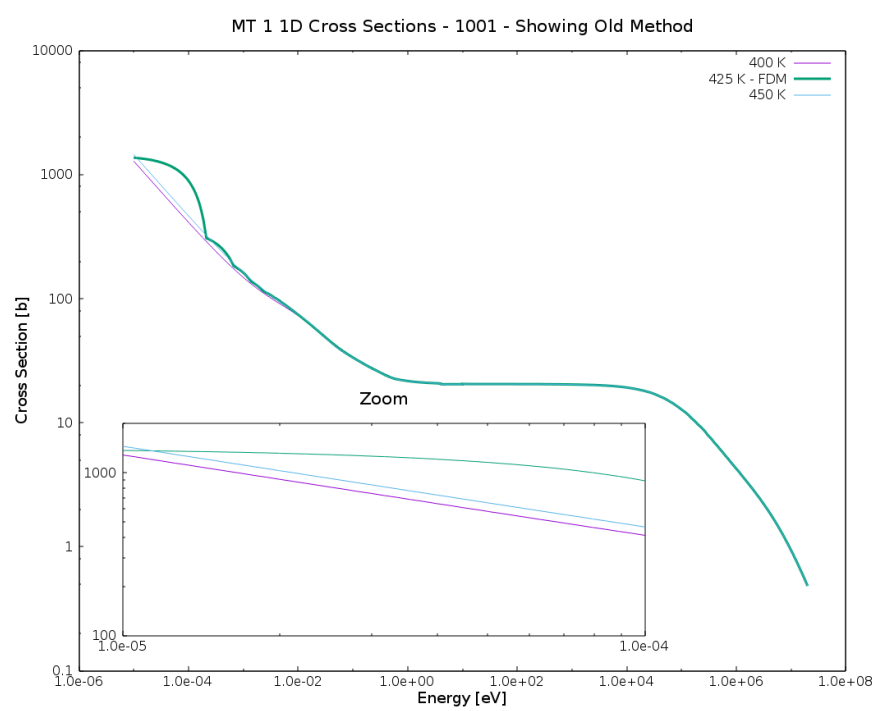

(a) Finite-difference method.

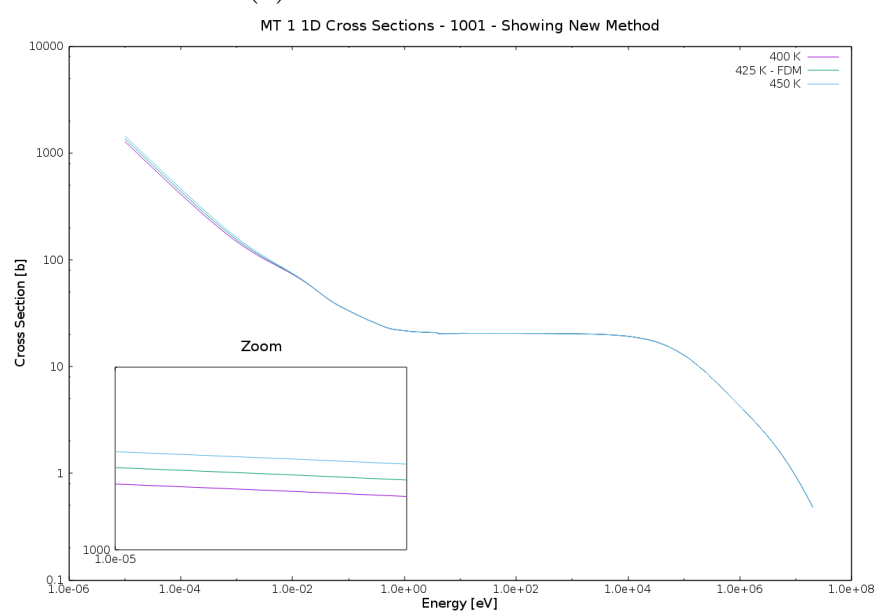

(b) Interpolation method.

Figure 3: Differences in ${ }^{1} \mathrm{H}$ cross sections.

Linear interpolation in the square root of temperature over the entire energy range can be used to remedy this problem. This should not introduce error due to the lack of resonances. Results from this interpolation are shown in Fig. 3b. Since this approach corrects the problem, interpolation should be used instead of the finite difference method for Doppler-broadening the one-dimensional cross sections for isotopes that have no resonance structure. If the cross sections are obtained from the ENDF thermal scattering sublibrary only interpolation is used.

\section{One-Dimensional Results}

A variety of test cases were run to test the impact of the one-dimensional problem-dependent Doppler broadening.

\subsection{Pin Cell}

The first case used a slightly enriched $(3.5 \%){ }^{235} \mathrm{U}$ pin cell. Before problem-dependent Doppler broadening was 
implemented, KENO would simply use cross sections at the closest temperature that was present in the libraries with no interpolation. If the user specified $760 \mathrm{~K}$, KENO would use $900 \mathrm{~K}$. As shown in Fig. 4, this leads to nonphysical "steps" in the eigenvalue as the library changes to another temperature. KENO was run with the problemdependent Doppler broadening enabled (titled CE-OTF), which shows a much smoother and more accurate eigenvalue curve as the temperature of the fuel increases.

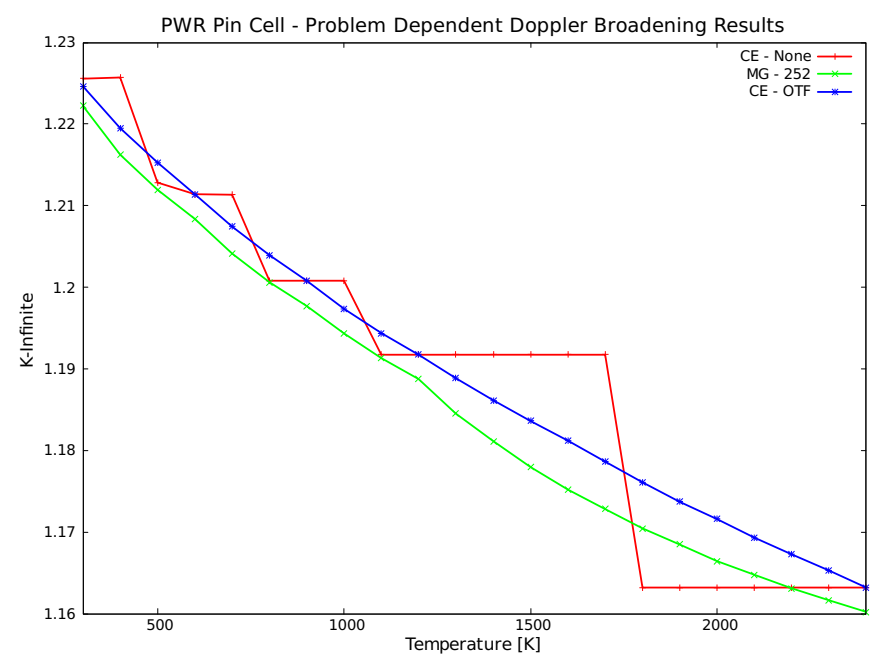

Figure 4: Results for a PWR pin cell showing impact of problemdependent Doppler broadening.

The multigroup 252 group library results are also shown in Fig. 4. Currently multigroup KENO uses linear interpolation to temperature-adjust multigroup libraries, which explains why it produces non-step-like eigenvalue estimates that show good agreement with the CE results when Doppleso broadening is enabled. Current reference libraries that ship with KENO include the temperatures $293 \mathrm{~K}, 565 \mathrm{~K}$, $600 \mathrm{~K}, 900 \mathrm{~K}, 1200 \mathrm{~K}$, and $2400 \mathrm{~K}$. KENO broadens from the closest, lower, cross section data file available. When the user requests a temperature that already exists on the ${ }_{185}$ provided library, KENO will not perform Doppler broadening. In Fig. 4 this manifests as the Doppler broadening results converging with the reference results at library temperatures.

\subsection{Radial and Axial Temperature Effects}

In order to better understand the effect of radial and axial temperature profiles in a fuel pin, two additional pin cell models were created. These pin cells were very similar to the one described previously, but one had nine radial temperature regions and the other had nine radial temperof 81 different temperature regions). These pin cells were designed to more closely mimic the temperature profile found in an operating reactor.

Fig. 5 shows the radial temperature used in the first problem-dependent Doppler broadening.

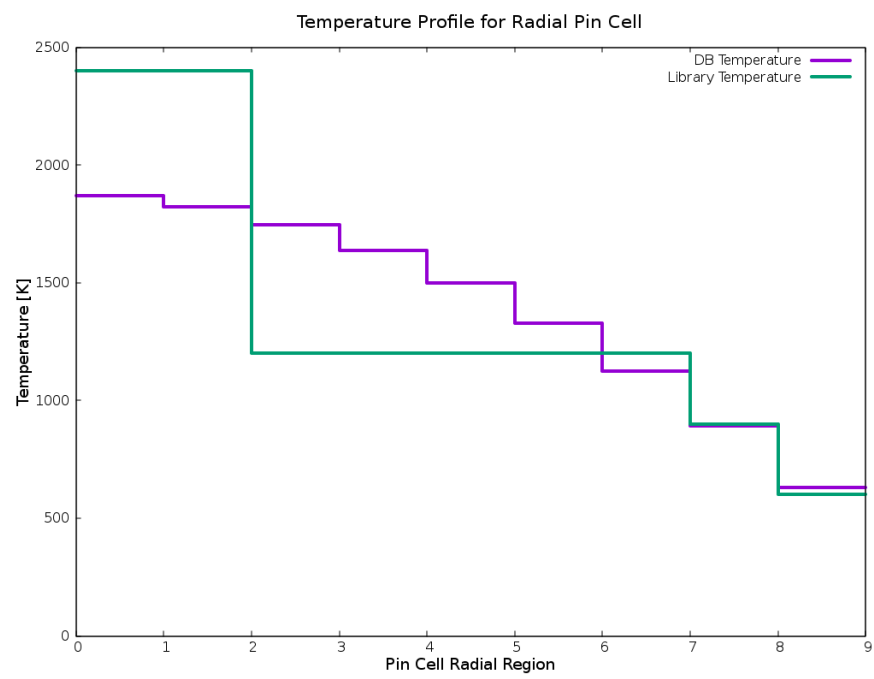

Figure 5: Radial temperature for the fuel pin model.

The impact of Doppler broadening on the eigenvalue is shown in Table 1, where $\sigma$ is the statistical uncertainty of k-effective from the Monte Carlo run.

Table 1: Eigenvalue results for pin cell with different radial temperatures.

\begin{tabular}{cccc}
\hline & No DB & DB & Difference $[\mathrm{pcm}]$ \\
\hline Eigenvalue & 1.31489 & 1.31260 & -174 \\
$(\sigma)$ & $(0.00015)$ & $(0.00014)$ & \\
\hline
\end{tabular}

For the case where both the axial and radial temperatures vary, the temperature profiles in both the axial and radial directions are shown in Fig. 6. Axial region 1 is the center axial region of the fuel pin, with axial region 2 being the region directly above and below it. Eigenvalue results are shown in Table 2 .

For both cases, the overall impact is to lower the eigenvalue. Fig. 4 shows that this is the expected behavior. As the temperature in the pin cell increases, the eigenvalue decreases. Since the net effect of the radial and axial proeigenvalue should be lower than that of the non-Dopplerbroadened case.

Table 2: Eigenvalue results for a pin cell with different axial and radial temperatures.

\begin{tabular}{cccc}
\hline & No DB & DB & Difference [pcm] \\
\hline $\begin{array}{c}\text { Eigenvalue } \\
(\sigma)\end{array}$ & 1.31623 & 1.31387 & -179 \\
\hline
\end{tabular}




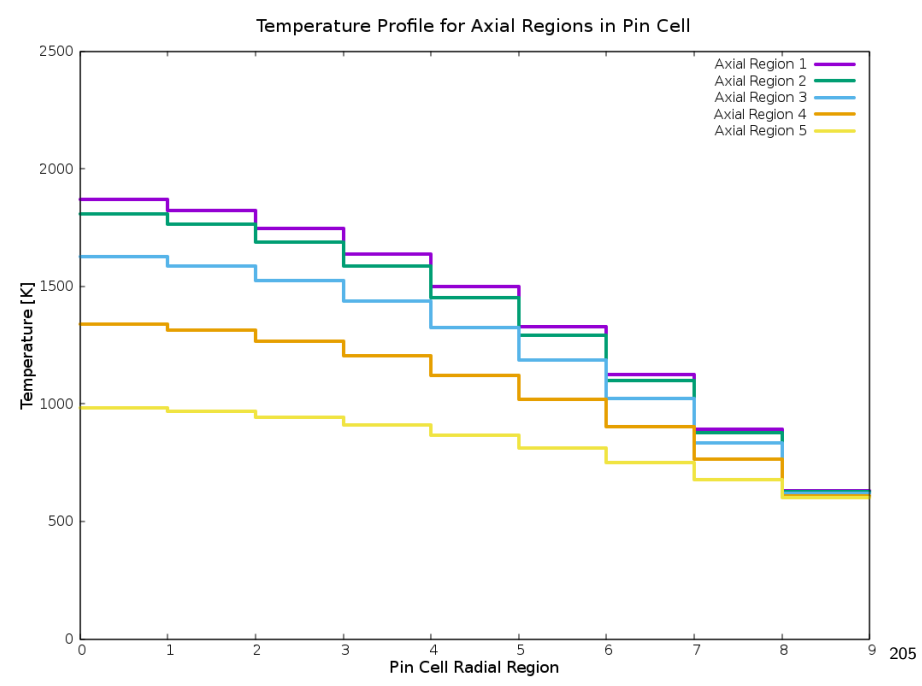

Figure 6: Radial temperature for each axial region of a pin cell.

\section{3. $3 D$ Assembly}

The next test case used is that of the full 3D assembly shown in Fig. 7 [17. The fuel assembly used lowenriched uranium and had 18 different temperatures along the length of the fuel rods. The large number of fuel regions with different temperatures will highlight the effects of Doppler broadening on the cross sections. Traditional KENO calculations would use the same cross sections for all 18 temperatures because all temperatures are near the same reference library, which, in this case, results in a 193 pcm difference in reactivity.

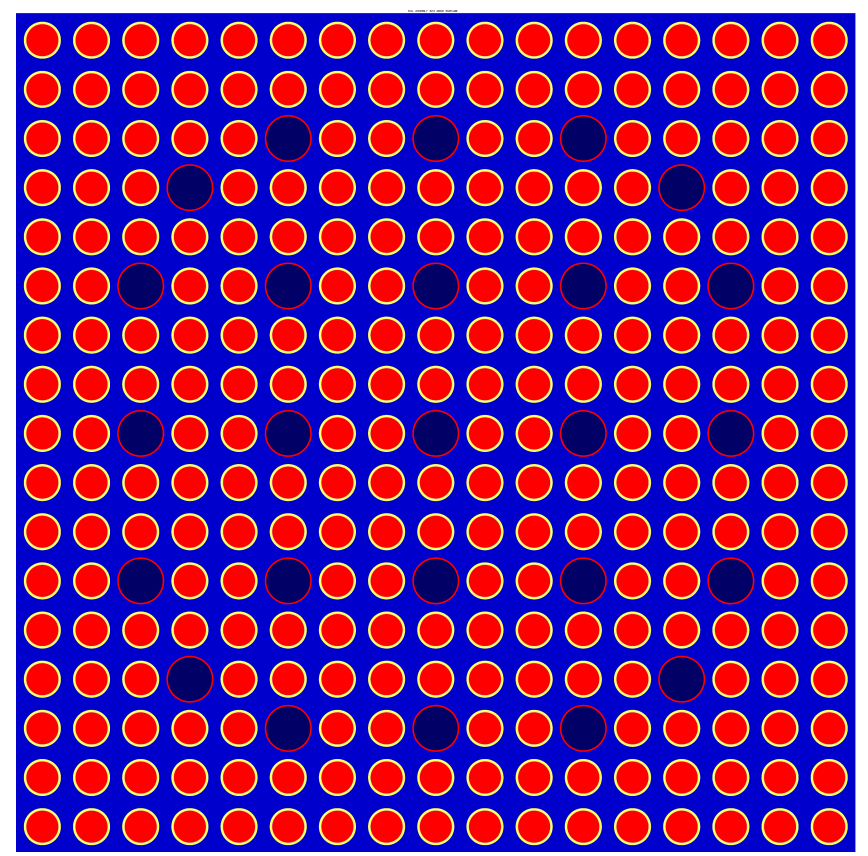

Figure 7: Radial view of a 3D assembly model generated by KENO. 225
Table 3: 3D assembly-problem-dependent Doppler-broadening results.

\begin{tabular}{ccc}
\hline Case & $\begin{array}{c}\text { Eigenvalue } \\
(\sigma)\end{array}$ & $\begin{array}{c}\text { Difference vs. } \\
\text { Multi-Group }\end{array}$ \\
\hline MG & 1.04323 & - \\
& $(0.00003)$ & \\
CE No DB & 1.04617 & 282 \\
& $(0.00005)$ & \\
CE w/ DB & 1.04424 & 97 \\
& $(0.00005)$ & \\
\hline
\end{tabular}

As seen in Table 3, using Doppler-broadened cross sections induces a difference of several hundred pcm. The impact on the run time is minimal because all cross sections are Doppler-broadened before any transport calculations are done. Cross sections would have to be recalculated any time the temperature changed, making ther- mohydraulic coupling inefficient. Most isotopes can be Doppler-broadened in a few seconds, and the worst-case scenario of ${ }^{238} \mathrm{U}$ takes less than $1 \mathrm{~min}$.

\section{Two-Dimensional Method}

After the one-dimensional cross sections have been Dopplerbroaded, the next step is to Doppler-broaden the twodimensional cross sections (or kinematics data) used for the thermal moderators.

\subsection{Interpolation Method on Double Differential Probabil- ity Data}

KENO uses cumulative probability distribution functions (CDFs) and probability distribution functions (PDFs) of the double differential scattering cross sections. The PDFs and CDFs are stored on an AMPX Continuous Energy (CE) library for use by KENO. During problem setup, will open the CE library with the closest temperature to the model temperature and will use the data for all neutron interactions with the thermal moderator.

The method implemented in KENO uses linear interpolation on the cosine $(\mu)$ and energy $(E)$ probabilities. This is different than previous methods done using on-the-fly sampling of the thermal neutron scattering data.18 For interpolation on cosines, no extra manipulation needs to be done, and the interpolated probability can be determined by

$$
\begin{aligned}
& p\left(\mu \mid E_{i n}, T\right)=p\left(\mu \mid E_{i n}, T_{i}\right)+ \\
& \frac{T-T_{i}}{T_{i+1}-T_{i}}\left[p\left(\mu \mid E_{i n}, T_{i+1}\right)-p\left(\mu \mid E_{i n}, T_{i}\right)\right],
\end{aligned}
$$

where $E_{\text {in }}$ is the incident energy of the neutron. For the outgoing energy $(E)$ probabilities, a traditional unit-base 
normalization 19 is applied before linear interpolation is done. The equation is then the same as Eq. (4):

$$
\begin{aligned}
& p\left(\hat{E} \mid \mu, E_{i n}, T\right)=p\left(\hat{E} \mid \mu, E_{i n}, T_{i}\right)+ \\
& \quad \frac{T-T_{i}}{T_{i+1}-T_{i}}\left[p\left(\hat{E} \mid \mu, E_{i n}, T_{i+1}\right)-p\left(\hat{E} \mid \mu, E_{i n}, T_{i}\right)\right] .
\end{aligned}
$$

Typically the interpolation is done on one of the CDF or PDF, and then the other value is recalculated.

The unit-base normalization is required when the energy range of a probability distribution varies at different $_{260}$ temperatures or incoming energies. Looking at Fig. 8, we can observe the major problem with Cartesian interpolation; the panel at $E$ will have features from the lower panel at the low end and from the upper panel at the high end. This will cause the resulting function to have arti- ${ }_{265}$ ficial peaks when the distributions shift as a function of energy, as is usually the case.

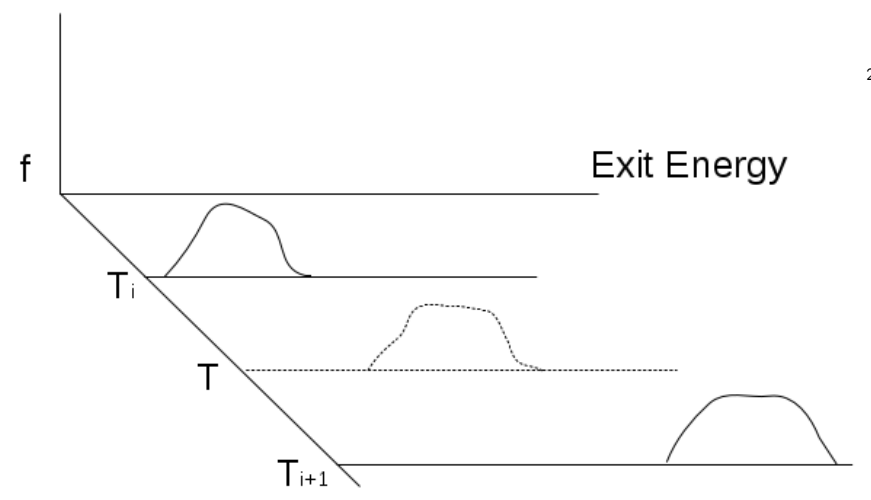

Temperatures

Figure 8: Interpolation between two-dimensional panels.

Eqs. (4) and (5) are relatively simple; however, a lot of effort has to go into ensuring that the interpolation is done on the same $E_{i n}$ and $\mu$ grid. Much of the coding is involved in dealing with this "bookkeeping." Generally, this is more difficult for the exit energy probabilities, as there the $\mu$ and $E_{i n}$ grids have to be the same. For those cases, the $\mu$ and $E_{\text {in }}$ grid from the closest temperature is used as a reference, and a "dummy" panel is created at the desired angles and energies for the far temperature using (unit-base) linear interpolation. Eq. (5) can then ${ }_{275}$ be used to calculate the probabilities at the intermediate temperature.

For the case where the cosine probabilities are equiprobable (generally the case for incoherent inelastic scattering), it is beneficial to do linear interpolation on the cosines instead of on the cosine CDFs and PDFs. This is done by ${ }_{280}$ using linear interpolation in temperature:

$$
\mu=\mu_{i}+\frac{T-T_{i}}{T_{i+1}-T_{i}}\left(\mu_{i+1}-\mu_{i}\right)
$$

\subsection{Two-Dimensional Results}

In order to test the Doppler broadening of the kinematic two-dimensional data, a variety of moderators have to be used. Some moderators will only have incoherent inelastic scattering, but some moderators will also have either incoherent or coherent elastic scattering. The Dopplerbroadening routines need to be able to handle all such cases successfully.

\subsubsection{Incoherent Inelastic Scattering}

Incoherent inelastic scattering (stored exclusively in MT 1007) is present in all thermal moderators and is arguably the most important scattering method present in the cross-section libraries. While any isotope would test this successfully, hydrogen in water was chosen as the main testing material. Hydrogen in water is the thermal moderator for the vast majority of operating nuclear reactors. As such, it is important to see if the interpolation scheme is producing reasonable results.

For hydrogen in water, data exist in the SCALE/ENDF cross-section libraries at the temperatures $293 \mathrm{~K}, 350 \mathrm{~K}$, $400 \mathrm{~K}, 450 \mathrm{~K}, 500 \mathrm{~K}, 550 \mathrm{~K}, 600 \mathrm{~K}, 650 \mathrm{~K}$, and $800 \mathrm{~K}$. Interpolation is controlled by the dbx parameter in KENO. By setting dbx to 2, two-dimensional interpolation is enabled. Results from these cases are shown in Fig. 9. As

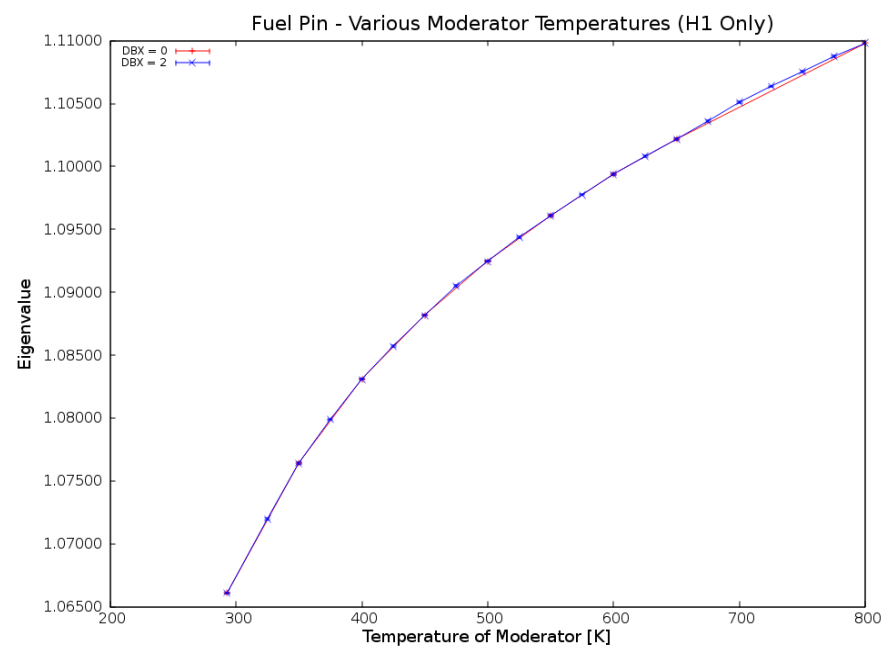

Figure 9: 2D interpolation results from pin cell (hydrogen in water).

can be seen from Fig. 9, results from the interpolated runs fall into the expected range of k-effective. There is some disagreement between $650 \mathrm{~K}$ and $800 \mathrm{~K}$ eigenvalues, but this is expected due to the large difference in temperature between the two reference libraries.

\subsubsection{Coherent Elastic Scattering}

Coherent elastic scattering is present in many crystalline materials and is characterized by the presence of Bragg edges. Bragg edges result in a histogram style of PDF (see Fig. 10), where neutrons will scatter at discrete angles. Elastic scattering is stored in MT 1008, and this 


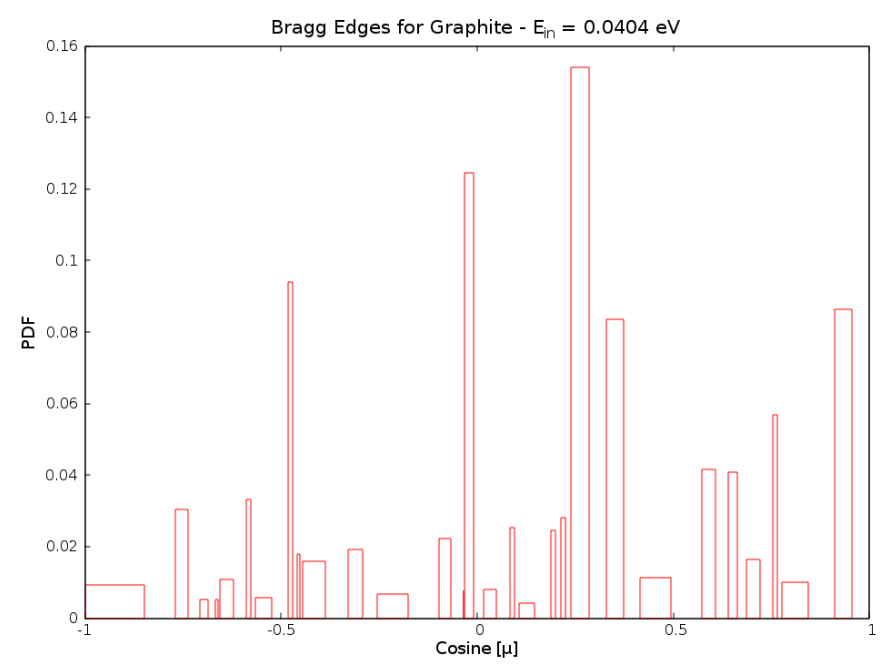

Figure 10: Bragg edges in graphite.

histogram nature is one easy way to test whether or not the scattering stored in MT 1008 is coherent or incoherent.

Interpolating the Bragg edge-scattering data is rela- ${ }^{-325}$ tively easy. If a specific scattering cosine exists at both temperatures, the new PDF can be interpolated between the two temperatures. If the specific scattering cosine only exists on one of the two temperatures, then the PDF is obtained by interpolating between the temperature where it and zero. In this way, the final results will contain the aggregate of the PDFs from both temperatures. The CDFs are then recalculated from the PDF data.

To test interpolation on this type of scattering, graphite is used as a moderator. The case tested involved a sphere uranium surrounded by a larger sphere of the graphite moderator. The fuel was slightly enriched uranium oxide $\left(\mathrm{UO}_{2}\right)$ at $900 \mathrm{~K}$. The graphite temperature was varied to obtain the results. Results from the current reference temperatures and from using interpolation are shown in Fig. 11. The eigenvalues resulting from the interpolation runs are consistent with what is expected by interpolating the eigenvalues from the reference library runs.

\subsubsection{Incoherent Elastic Scattering}

Incoherent elastic scattering is stored much like incoherent inelastic scattering, except that it is stored in MT 1008 and does not generally use equiprobable cosine bins. Therefore it can be treated much the same as inelastic scattering, except allowances have to be made to allow for ${ }_{33}$ cosines to exist on some temperatures and not others. An isotope will not have both incoherent and coherent elastic scattering. Therefore, MT 1008 can be used for both types of scattering.

The test case used for incoherent elastic scattering was hydrogen in polyethylene. Like all thermal moderators, ${ }^{335}$ hydrogen in polyethylene contains incoherent inelastic scattering in MT 1007; however it also contains incoherent

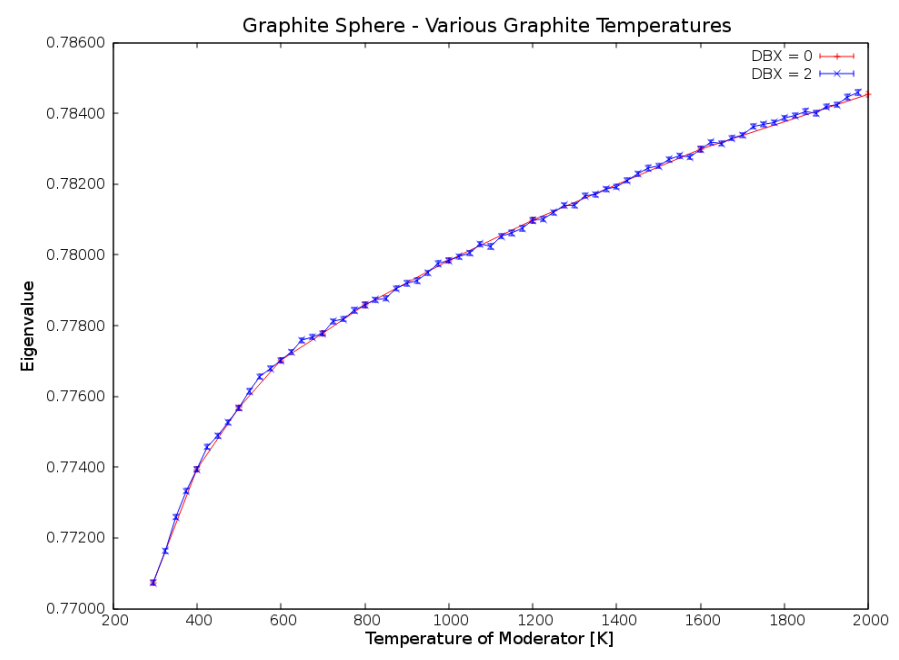

Figure 11: 2D interpolation results from graphite sphere.

elastic scattering in MT 1008. Unlike the other cases presented thus far, there are only two reference temperatures available for polyethylene: $293 \mathrm{~K}$ and $300 \mathrm{~K}$. Results from the current reference temperatures and from using interpolation are shown in Fig. 12. Again, the eigenvalues resulting from these interpolation runs are consistent with what is expected by interpolating the eigenvalues from the reference library runs.

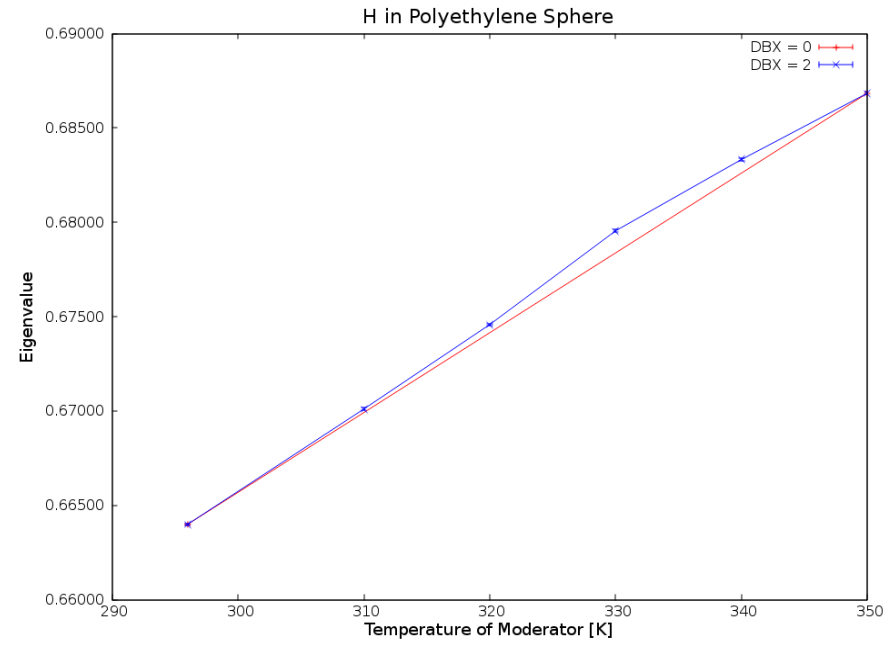

Figure 12: 2D interpolation results from polyethylene sphere.

\section{Benchmarks}

The International Handbook of Evaluated Reactor Physics Benchmark Experiments (IHERPhBE) [20] was prepared by a working group of experienced reactor physics personnel. It contains reactor physics benchmark specifications that have been derived from experiments performed at various nuclear experimental facilities around the world. The benchmark specifications are intended for use to validate calculation techniques. The most recent edition of 
the handbook contains data from 53 different experimental series that were performed at 31 different reactor facilities.

Many of the benchmarks in the handbooks were conducted at room temperature. Therefore one goal was to find several elevated-temperature benchmarks that could be used to test the effectiveness of the problem-dependent

345 Doppler-broadening methods. Several benchmarks were identified, all at elevated temperatures of around $500 \mathrm{~K}$. Since the closest cross-section libraries provided with SCALE are at $565 \mathrm{~K}$, it should provide a good check for the accuracy of the Doppler-broadening methods.

\subsection{KRITZ-2:19}

The first evaluation, known as KRITZ-2:19, was an experiment with mixed-oxide rods at an $18.00 \mathrm{~mm}$ pitch. Criticality was obtained by controlling the boron content in the water and by adjusting the water level. The square inner tank was filled with water up to the level needed to achieve criticality. The space between the inner tank and the cylindrical outer tank served as a dump area and was effectively filled with steam.

The experimental, measured, and benchmark-model $\mathrm{k}_{\text {effs }}$ are given in Table 4 As a critical reactor, it was expected that the measured and experimental $\mathrm{k}_{\mathrm{eff}}$ will be unity. Due to simplifications made in the model, it was determined that the benchmark $\mathrm{k}_{\text {eff }}$ will actually be slightly above unity. The benchmark $\mathrm{k}_{\text {eff }}$ was the value expected to be calculated with the Monte Carlo codes. Tables 5 and 6 show the benchmark results from MCNP (obtained from the evaluation report) and from KENO, respectively. MCNP used a weighted mixture of cross sections from the two closest temperature libraries to obtain cross sections that are accurate at the desired temperatures. KENO was fairly close to the MCNP results for the cold case when they are both using the ENDF 7.0 library. When the 7.1 library was used, the eigenvalue fell slightly but was still within two standard deviations of the benchmark. For the result only when Doppler broadening was enabled. This was expected since the temperature of the hot case is 510 $\mathrm{K}$, which is sufficiently far from the closest library $(565 \mathrm{~K})$ to introduce error.

\subsection{KRITZ-2:1}

The second KRITZ evaluation, known as KRITZ-2:1, was a rectangular array of low enriched uranium Zircalloy2 fuel rods in light water 21. Again, criticality was achieved by regulating the concentration of boron in water and by adjusting the water level.

The experimental, measured, and benchmark-model $\mathrm{k}_{\text {effs }}$ are given in Table 7 . The statements regarding the $\mathrm{k}_{\text {eff }}$ values made in regard to KRITZ-2:19 (Section 5.1) also apply for this KRITZ case. Tables 8 and 9 show the benchmark results from MCNP (obtained from the evaluation report) and from KENO for the cold and hot
Table 4: Experimental, measured, and benchmark-model $\mathrm{k}_{\mathrm{eff}}$ for KRITZ-2:19.

\begin{tabular}{ccc}
\hline & Cold & Hot \\
\hline Measured & $1.0000 \pm 0.0001$ & $1.0000 \pm 0.0001$ \\
Experimental & $1.0000 \pm 0.0015$ & $1.0000 \pm 0.0019$ \\
Benchmark & $1.0077 \pm 0.0030$ & $1.0055 \pm 0.0027$ \\
\hline
\end{tabular}

Table 5: Benchmark results for KRITZ-2:19 cold case.

\begin{tabular}{ccc}
\hline & $\mathrm{k}_{\text {eff }}$ & $\frac{C}{E}-1(\%)$ \\
\hline MCNP & $1.0023 \pm 0.0001$ & -0.54 \\
JEFF 3.1 & \\
MCNP & $1.0031 \pm 0.0001$ & -0.44 \\
ENDF/B-VII.0 & \\
KENO & & \\
ENDF/B-VII.0 & $1.0027 \pm 0.0001$ & -0.50 \\
DBX $=0$ & & \\
KENO & & \\
ENDF $/$ B-VII.1 & $1.0019 \pm 0.0001$ & -0.58 \\
DBX $=0$ & & \\
& & \\
\hline
\end{tabular}

Table 6: Benchmark results for KRITZ-2:19 hot case.

\begin{tabular}{ccc}
\hline & $\mathrm{k}_{\text {eff }}$ & $\frac{C}{E}-1(\%)$ \\
\hline MCNP & $1.0011 \pm 0.0001$ & -0.44 \\
JEFF 3.1 & \\
KENO & & \\
ENDF /B-VII.0 & $0.9991 \pm 0.0001$ & -0.64 \\
DBX $=0$ & & \\
KENO & & -0.45 \\
ENDF /B-VII.0 & $1.0010 \pm 0.0001$ & \\
DBX $=2$ & & -0.69 \\
KENO & & \\
ENDF $/$ B-VII.1 & $0.9986 \pm 0.0001$ & \\
DBX $=0$ & & -0.50 \\
KENO & & \\
ENDF $/ B-V I I .1$ & $1.0005 \pm 0.0001$ & \\
DBX $=2$ & & \\
\hline
\end{tabular}


cases, respectively. Again, MCNP used a weighted mixture of cross sections from the two closest libraries to obtain cross sections that are accurate at the desired temperatures. For the cold case, KENO was actually slightly closer to the benchmark values than MCNP. For the hot case, KENO was slightly further away from the benchmark than MCNP. Without Doppler broadening, the KENO results are particularly off the benchmark. Enabling Doppler broadening increased the $\mathrm{k}_{\mathrm{eff}}$ by $\sim 150 \mathrm{pcm}$ and made the results closer to the benchmark. Like previous cases, enabling direct $S(\alpha, \beta)$ lowered the $\mathrm{k}_{\mathrm{eff}}$ by $\sim 10 \mathrm{pcm}$, which was generally within one standard deviation of the original KENO result.

\section{Conclusions}

Typically, reactor analysis tools ship with only a subset of temperatures that a reactor physics analyst needs in order to accurately model a problem. A one-dimensional method to Doppler-broaden cross secions was expanded and coded into the Monte Carlo code KENO. By using this method, cross sections can be Doppler-broadened to any temperature that the user selects. Doppler broadening is done before neutron transport begins. Therefore the method has been christened as "problem-dependent Doppler pre-broadening." Using the temperature-corrected data shows an immediate impact when a temperature that is not in a library is used. Having correct temperatures for each fuel and moderator region will become increasingly important as Monte Carlo is used to model full reactors. It is also a paramount ability if accurate coupling to thermal-hydraulic codes is desired.

In addition to Doppler broadening the one-dimensional cross sections, correcting the temperature of the twodimensional kinematics data was also examined. A unitbase interpolation sheme was devised and implemented for the energy and scattering cosine cumulative distribution functions. It was shown that, by using this method, more accurate results could be obtained at temperatures between those provided by the libraries.

A variety of benchmarks taken from reactor physics and criticality safety benchmark evaluations were used to verify the accuracy of the new methods. Efforts were made to find evaluations that contained cases that were run at an elevated temperature in order to test the effectiveness of the Doppler-broadening mechanisms. Overall, the results became more accurate when the Doppler-broadening methods were used. In the future, as model complexity and multiphysics couplings increase, it will become more and more important to have an accurate cross-section and

\section{Acknowledgements}

The work documented in this paper was performed with support from the U.S. Department of Energy Nuclear Criticality Safety Program.

Table 7: Experimental, measured, and benchmark-model $\mathrm{k}_{\mathrm{eff}}$ for KRITZ-2:1.

\begin{tabular}{ccc}
\hline & Cold & Hot \\
\hline Measured & $1.0000 \pm 0.0001$ & $1.0000 \pm 0.0001$ \\
Experimental & $1.0000 \pm 0.0018$ & $1.0000 \pm 0.0026$ \\
Benchmark & $1.0025 \pm 0.0020$ & $1.0024 \pm 0.0028$ \\
\hline
\end{tabular}

Table 8: Benchmark results for KRITZ-2:1 cold case.

\begin{tabular}{ccc}
\hline & $\mathrm{k}_{\text {eff }}$ & $\frac{C}{E}-1(\%)$ \\
\hline MCNP & $0.9975 \pm 0.0001$ & -0.50 \\
JEFF 3.1 & \\
MCNP & $0.9981 \pm 0.0001$ & -0.43 \\
ENDF/B-VII.0 & \\
KENO & & \\
ENDF/B-VII.0 & $0.9990 \pm 0.0001$ & -0.35 \\
DBX $=0$ & & \\
\hline
\end{tabular}

Table 9: Benchmark results for KRITZ-2:1 hot case.

\begin{tabular}{ccc}
\hline & $\mathrm{k}_{\text {eff }}$ & $\frac{C}{E}-1(\%)$ \\
\hline MCNP & $0.9992 \pm 0.0001$ & -0.33 \\
JEFF 3.1 & \\
KENO & \\
ENDF/B-VII.0 & $0.9958 \pm 0.0001$ & -0.66 \\
DBX =0 & \\
KENO & \\
ENDF/B-VII.0 & $0.9973 \pm 0.0001$ & -0.51 \\
DBX $=2$ & & \\
& & \\
\hline
\end{tabular}


[1] A. Trkov, M. Herman, D. Brown, ENDF-6 Formats Manual: Data Formats and Procedures for the Evaluated Nuclear Data Files ENDF/B-VI and ENDF/B-VII, Tech. rep., CSEWG Doc- ${ }_{520}$ ument ENDF-102, Report BNL-90365-2009 Rev. 2, Brookhaven National Laboratory, Upton, New York (2011).

[2] M. Dunn, N. Greene, AMPX-2000: A cross-section processing system for generating nuclear data for criticality safety applications, Trans. Am. Nucl. Soc 86 (2002) 118-119.

[3] R. E. MacFarlane, D. W. Muir, NJOY99.0 - Code System for ${ }^{525}$ Producing Pointwise and Multigroup Neutron and Photon Cross Sections from ENDF/B Data, Los Alamos, New Mexico, pSR480/NJOY99.00 (2000).

[4] D. Hollenbach, L. Petrie, S. Goluoglu, N. Landers, M. Dunn, KENO-VI: A general quadratic version of the keno program, Tech. rep., Oak Ridge National Laboratory, Oak Ridge, TN (2011).

[5] D. E. Cullen, O. Ozer, C. R. Weisbin, Exact Doppler broadening of evaluated neutron cross sections, in: Trans. Am. Nuc. Soc., Vol. 16, ANS, 1973, p. 320.

[6] ORNL, Scale: A Comphrehensive Modeling and Simulation Suite for Nuclear Safety Analysis and Design, Oak Ridge National Laboratory, Oak Ridge, Tennessee, oRNL/TM-2005/39 (2011).

[7] G. Yesilyurt, W. R. Martin, F. Brown, On-The-Fly Doppler Broadening for Monte Carlo Codes, in: International Conference on Mathematics, Computational Methods \& Reactor Physics, ANS, Saratoga Springs, New York, 2009, may 3-7.

[8] G. Yesilyurt, W. R. Martin, F. B. Brown, On-the-Fly Doppler Broadening for Monte Carlo Codes, Nuclear Science and Engineering 171 (3) (2012) 239-257.

[9] F. B. Brown, W. R. Martin, G. Yesilyurt, S. Wilderman, Progress with On-The-Fly Neutron Doppler Broadening in MCNP, in: Transactions of the American Nuclear Society, ANS, Chicago, Illinois, 2012, june 24-28.

[10] W. R. Martin, S. Wilderman, F. B. Brown, G. Yesilyurt, Implementation of On-the-Fly Doppler Broadening in MCNP, in: International Conference on Mathematics and Computational Methods Applied to Nuclear Science \& Engineering, ANS, Sun Valley, Idaho, 2013, may 5-9.

[11] T. H. Trumbull, Treatment of Nuclear Data for Transport Problems Containing Detailed Temperature Distributions, Nuclear Technology 156 (1) (2006) 75-86.

[12] X-5 Monte Carlo Team, MCNP-a general purpose monte carlo n-particle transport code, Tech. Rep. LA-UR-03-1987, Los Alamos National Laboratory, version 5 (April 2003).

[13] J. Walsh, B. Forget, K. Smith, B. Kiedrowski, F. Brown, Direct, on-the-fly calculation of unresolved resonance region cross sections in monte carlo simulations, in: Proc. ANS MC2015, 2015.

495 [14] S. Hart, G. I. Maldonado, C. Celik, L. C. Leal, Problemdependent doppler broadening of continuous-energy cross section in the KENO Monte Carlo code, in: PHYSOR 2014, American Nuclear Society, Kyoto, Japan, 2014.

[15] N. M. Larson, Updated Users' Guide for SAMMY: Multilevel R-Matrix Fits to Neutron Data Using Bayes' Equations, Oak Ridge National Laboratory, oRNL/TM-9179/R8 (2008).

[16] L. C. Leal, R. Hwang, A Finite Difference Method for Treating the Doppler Broadening of Neutron Cross Sections, in: American Nuclear Society, Los Angles, CA, 1987.

[17] B. T. Rearden, W. J. Marshall, C. M. Perfetti, J. Miss, Y. Richet, Quantifying the effect of undersampling biases in Monte Carlo reaction rate tallies, Tech. rep., Organisation for Economic Cooperation and Development Nuclear Energy Agency Expert Group on Advanced Monte Carlo Techniques Benchmark Proposal (2013).

[18] A. T. Pavlou, W. Ji, On-the-fly sampling of temperaturedependent thermal neutron scattering data for monte carlo simulations, Annals of Nuclear Energy 71 (2014) 411-426.

[19] R. J. Doyas, S. T. Perkins, Interpolation of tabular secondary neutron and photon energy distributions Nucl. Sci. Eng 50
(1973) 390-392

URL http://www . ans .org/pubs/journals/nse/a_26575

[20] N. N. S. Committee, et al., International handbook of evaluated reactor physics benchmark experiments, Tech. rep., NEA/NSC/DOC (2006).

[21] L. Snoj, J. C. Gehin, KRITZ-2:1 Experiment on Regular $\mathrm{H}_{2} \mathrm{O} /$ Fuel Pin Lattices with Low Enriched Uranium Fuel at Temperatures $248.5{ }^{\circ} \mathrm{C}$, Tech. Rep. KRITZ-LWR-RESR-002, Jozef Sefan Institute and Oak Ridge National Laboratory (March 2009). 